\title{
A Bayesian Framework for Reliability Assessment via Wiener Process and MCMC
}

\author{
Huibing Hao ${ }^{1,2}$ and Chun Su${ }^{1}$ \\ ${ }^{1}$ Department of Industrial Engineering, Southeast University, Nanjing 211189, China \\ ${ }^{2}$ Department of Mathematics, Hubei Engineering University, Xiaogan 432100, China \\ Correspondence should be addressed to Huibing Hao; haohuibing1979@126.com
}

Received 27 August 2013; Revised 2 March 2014; Accepted 14 March 2014; Published 9 April 2014

Academic Editor: Sarp Adali

Copyright (C) 2014 H. Hao and C. Su. This is an open access article distributed under the Creative Commons Attribution License, which permits unrestricted use, distribution, and reproduction in any medium, provided the original work is properly cited.

The population and individual reliability assessment are discussed, and a Bayesian framework is proposed to integrate the population degradation information and individual degradation data. Different from fixed effect Wiener process modeling, the population degradation path is characterized by a random effect Wiener process, and the model can capture sources of uncertainty including unit to unit variation and time correlated structure. Considering that the model is so complicated and analytically intractable, Markov Chain Monte Carlo (MCMC) method is used to estimate the unknown parameters in the population model. To achieve individual reliability assessment, we exploit a Bayesian updating method, by which the unknown parameters are updated iteratively. Based on updated results, the residual use life and reliability evaluation are obtained. A lasers data example is given to demonstrate the usefulness and validity of the proposed model and method.

\section{Introduction}

Due to the advances in material science and manufacturing processes, most modern products have long lifetimes and high reliability, and few units will fail in a test of practical length at normal operating conditions [1]. Therefore, it is difficult to assess the reliability of these products with traditional failure time method. In such cases, degradation data can be used as an alternate resource for reliability analysis from an economical and practical viewpoint [2]. In the last decades, degradation data has played an important role in reliability assessment.

Degradation, such as wear, erosion, and fatigue, is very common for most mechanical systems or components. It can be described by a continuous performance process in terms of time [3, 4]. Reference [3] has presented three kinds of methods for degradation data analysis, including linear regression method, degradation path method, and stochastic process method. Considering that stochastic process can provide a flexible way to describe the failure mechanism and characteristics of operating environment, it has been widely used to model the degradation path, such as Markov Chain [5], Gamma process [6,7], and Wiener process [8-14].
Among those stochastic processes, Wiener process has become very popular for degradation modeling in recent years, such that Tseng et al. [8] and Tang and Su [9] utilized Wiener process to describe the lifetime for LED lamps and Lee and Tang [10] handled the failure time prediction problem based on Wiener process with drift under a time censored degradation test. Park and Padgett [11] used Wiener process to model the initial damage under accelerated testing. Considering that each item possibly experiences different sources of variations during its operation, for a degradation model to be realistic, it is more appropriate to incorporate unit to unit variability in the degradation process. But the above degradation models do not take into account.

Recently, Peng and Tseng [12] incorporated the random effect in the drift coefficient and measurement errors in Wiener process for lifetime assessment. Wang [13] extended the Wiener process model further to include both a random effect and a nondecreasing transformation of the time scale. Si et al. [14] considered Wiener process using a mixed coefficient model to obtain an approximation probability density function (PDF) of the residual use life (RUL). However, the proposed estimation procedure only utilized the current degradation data without considering the history 
degradation information up to date, and then Bayesian analysis approach is used. Gebraeel et al. [15] considered the exponential degradation model and developed a Bayesian approach to update the unknown parameters. Then, the approach was further developed [16, 17]. But in their papers, they only considered single unit history information.

In this paper, a general random effect Wiener process model is proposed to characterize the population degradation path, and the random effect model can describe the unit to unit variation of the product and the time correlated structure. The model in such a situation is very complicated and becomes cumbersome from a computational viewpoint. For this reason, the Bayesian Markov Chain Monte Carlo (MCMC) method is used to obtain the unknown parameters [18]. In individual reliability assessment, a Bayesian framework is proposed to integrate the population degradation information and the individual degradation data, and the reliability assessment of particular individual and its residual use life can be obtained.

The rest of the paper is organized as follows. In Section 2, some degradation models and selection criteria are described. Then, the population degradation modeling and parameters estimation method are introduced in Section 3. Section 4 presents technical details on how to update the individual degradation model parameters and obtain the reliability assessment of a particular individual unit. A numerical example about lasers data is given in Section 5. Finally, some conclusions are made in Section 6.

\section{Degradation Model and Selection Criteria}

2.1. Degradation Model. A well-adopted form for the Wiener process $\{X(t), t \geq 0\}$ can be expressed as $M_{1}$ :

$$
X(t)=\mu t+\sigma B(t)
$$

where $B(t)$ is the standard Brownian motion representing a time correlated structure and $\mu$ and $\sigma$ are the unknown drift and diffusion parameters, respectively.

In most cases, each unit usually experiences different sources of variations during their operation. For a degradation model to be realistic, it is more appropriate to incorporate unit to unit variability in the degradation process. In this paper, the drift parameter $\mu$ and diffusion $\sigma$ are regarded as random variables. The degradation model is specified as $M_{2}$ :

$$
\begin{aligned}
& X(t)=\mu t+\sigma B(t), \\
& v=\sigma^{-2} \sim G(\beta, \alpha), \\
& \mu \mid v \sim N\left(\theta, \frac{\lambda}{v}\right),
\end{aligned}
$$

where $\beta, \alpha, \theta$, and $\lambda$ are unknown parameters and $G(\cdot)$, and $N(\cdot, \cdot)$ are Gamma distribution and normal distribution, respectively. The advantages of the degradation model $M_{2}$ allow us to take unit to unit variation and time correlated structure into considerations simultaneously.
Note that if the diffusion coefficient $\sigma$ is a constant and does not change with time, then the degradation model $M_{2}$ reduces to the conventional mixed effect model $M_{3}$ :

$$
\begin{gathered}
X(t)=\mu t+\sigma B(t), \\
\mu \sim N\left(\eta, \sigma_{\eta}^{2}\right),
\end{gathered}
$$

where $\eta, \sigma_{\eta}$, and $\sigma$ are unknown parameters.

2.2. Model Selection Criteria. The performance of a degradation model strongly depends on the appropriateness modeling of a product's degradation path. When some alternative models are proposed, it is interesting to compare their relative performances. Spiegelhalter et al. [19] proposed the deviance information criterion (DIC), and this criterion is specifically useful for selection models under the Bayesian approach where the parameters of the model are obtained by using MCMC method.

The deviance is defined by

$$
D(\delta)=-2 \log L(\delta),
$$

where $\delta$ is a vector of unknown parameters and $L(\delta)$ is the likelihood function.

Then, the DIC is defined by

$$
\mathrm{DIC}=D(\widehat{\delta})+2 n_{D},
$$

where $D(\widehat{\delta})$ is the deviance evaluated at the posterior mean $\widehat{\delta}=E(\delta \mid$ data $)$ and $n_{D}$ is the effective number of parameters given by $n_{D}=\bar{D}-D(\widehat{\delta})$, where $\bar{D}=E(D(\delta) \mid$ data $)$ is the posterior deviance measuring the quality of the data fit for the model. Smaller value of DIC indicates better model. Note that these values can be negative (see [20]).

\section{Reliability Assessment of Population Degradation Model}

In this section, the random effect Wiener process model is used to characterize the population degradation path, and the reliability assessment of population can characterize the average survival ability of the population. Considering that the model is very complicated, the MCMC method is used to obtain the unknown parameter estimation.

3.1. Population Reliability Modeling. Let $\xi$ be the threshold value of the product, and the product's lifetime $T$ is defined as

$$
T=\inf \{t \mid X(t) \geq \xi\}
$$

Assume that the degradation path of a product is governed by the model $M_{1}$. It is known that $T$ follows inverse Gaussian distribution with PDF as

$$
f_{T}(t \mid \mu, v)=\frac{\xi}{\sqrt{2 \pi v^{-1} t^{3}}} \exp \left(-\frac{v(\xi-\mu t)^{2}}{2 t}\right) .
$$


Based on the PDF of the lifetime $T$, the reliability at time $t$ can be expressed as

$$
\begin{aligned}
R(t) & =\operatorname{Pr}(T>t)=\int_{t}^{+\infty} f_{T}(x) d x \\
& =\Phi\left(-\frac{\mu t-\xi}{\sqrt{v^{-1} t}}\right)-\exp \left(\frac{2 \mu \xi}{v^{-1}}\right) \Phi\left(-\frac{\mu t+\xi}{\sqrt{v^{-1} t}}\right),
\end{aligned}
$$

where $\Phi(\cdot)$ is the distribution function of the standard normal distribution.

When the diffusion coefficient $\sigma=v^{-1 / 2}$ is constant and the drift parameter $\mu$ is random variable, by using the total law of probability, the PDF of the lifetime $T$ can be reconstructed in model $M_{3}$ as

$$
\begin{aligned}
& f_{T}(t \mid v) \\
& \quad=\int_{-\infty}^{+\infty} f_{T}(t \mid \mu, v) \varphi\left(\frac{\mu-\eta}{\sigma_{\eta}}\right) d \mu \\
& \quad=\sqrt{\frac{\xi^{2}}{2 \pi\left(v^{-1}+\sigma_{\eta}^{2} t\right) t^{3}}} \exp \left(-\frac{(\xi-\eta t)^{2}}{2\left(v^{-1} t+\sigma_{\eta}^{2} t^{2}\right)}\right),
\end{aligned}
$$

where $\varphi(\cdot)$ is the distribution function of the standard normal distribution.

Then, the reliability at time $t$ can be expressed as

$$
\begin{aligned}
& R(t) \\
& =\Phi\left(-\frac{\eta t-\xi}{\sqrt{\sigma_{\eta}^{2} t^{2}+v^{-1} t}}\right) \\
& \quad-\exp \left(\frac{2 \eta v^{-1} \xi+2 \sigma_{\eta}^{2} \xi^{2}}{v^{-2}}\right) \Phi\left(-\frac{2 \sigma_{\eta}^{2} \xi t+v^{-1}(\eta t+\xi)}{v^{-1} \sqrt{\sigma_{\eta}^{2} t^{2}+v^{-1} t}}\right) .
\end{aligned}
$$

Similarly, when $\mu$ and $v$ are the random variables, by using the total law of probability, we can get the PDF of the lifetime $T$ in model $M_{2}$ as

$$
\begin{aligned}
& f_{T}(t) \\
& =\frac{\xi \alpha^{\beta}}{2 \pi \sqrt{\lambda t^{3}} \Gamma(\beta)} \\
& \times \int_{0}^{+\infty}\left\{v^{\beta-1}\right. \\
& \times \exp \left[-\left(\frac{(\xi-\theta t)^{2}}{2 t(1+\lambda t)}+\alpha\right) v\right. \\
& \times \int_{-\infty}^{+\infty} \exp \left[-\left(\mu-\frac{\lambda \xi+\theta}{1+\lambda t}\right)^{2}\right. \\
& \left.\left.\left.\times\left(\frac{2 \lambda}{(1+\lambda t) v}\right)^{-1}\right] d \mu\right]\right\} d v
\end{aligned}
$$

$$
\begin{aligned}
= & \frac{\xi \alpha^{\beta}}{\sqrt{2 \pi \lambda t^{3}(1+\lambda t)} \Gamma(\beta)} \\
& \times \int_{0}^{+\infty} \exp \left[-\left(\frac{(\xi-\theta t)^{2}}{2 t(1+\lambda t)}+\alpha\right) v\right] v^{\beta-(3 / 2)} d v \\
= & \frac{\Gamma(\beta+(1 / 2)) \xi}{\sqrt{2 \pi t^{3}[\alpha(\lambda t+1)]} \Gamma(\beta)}\left(1+\frac{(\xi-\theta t)^{2}}{2 \alpha\left(\lambda t^{2}+t\right)}\right)^{-\beta-(1 / 2)},
\end{aligned}
$$

and the reliability function is

$$
\begin{aligned}
R(t)=1-P(T \leq t) & \\
=1-\int_{0}^{t} & \frac{\Gamma(\beta+(1 / 2)) \xi}{\sqrt{2 \pi x^{3}[\alpha(\lambda x+1)]} \Gamma(\beta)} \\
& \quad \times\left(1+\frac{(\xi-\theta x)^{2}}{2 \alpha\left(\lambda x^{2}+x\right)}\right)^{-\beta-(1 / 2)} d x .
\end{aligned}
$$

3.2. Parameters Estimation via the MCMC Method. Suppose that the degradation path of product is governed by $M_{2}$; we know that the conditional distribution of $X(t)$ given by $\mu$ and $v$ is normal distribution, and then the marginal density of $X(t)$ follows

$$
\begin{aligned}
& f(x)= \frac{\alpha^{\beta}}{2 \pi \Gamma(\beta) \sqrt{\lambda}} \\
& \times \int_{-\infty}^{+\infty} \int_{0}^{+\infty} v^{\beta} \exp \left\{-\left(\frac{(x-\mu t)^{2}}{2}\right.\right. \\
&\left.\left.\quad+\frac{(\mu-\theta)^{2}}{2 \lambda}+\alpha\right) v\right\} d v d \mu \\
&=\frac{\Gamma(\beta+(1 / 2))}{\sqrt{2 \pi\left[\alpha\left(\lambda t^{2}+t\right)\right]} \Gamma(\beta)}\left(1+\frac{(x-\theta t)^{2}}{2 \alpha\left(\lambda t^{2}+t\right)}\right)^{-\beta-(1 / 2)} .
\end{aligned}
$$

Note that $\sqrt{\beta / \alpha\left(\lambda t^{2}+t\right)}(X(t)-\theta t)$ has a $T$ distribution with degrees of freedom $2 \beta$. That is to say,

$$
X(t) \sim T_{2 \beta}\left(\frac{\sqrt{\beta}(\theta t-\xi)}{\sqrt{\alpha\left(\lambda t^{2}+t\right)}}\right),
$$

where $T_{2 \beta}$ is the $T$ distribution function with degrees of freedom $2 \beta$.

To achieve parameters estimation, we assume that $n$ units are tested and $X_{i}\left(t_{i j}\right)$ denotes the cumulative degradation values of product $i$ at time $t_{i j}$, for $i=1,2, \ldots, n, j=$ $0,1,2, \ldots, m$.

Let

$$
\begin{gathered}
\Delta X_{i}\left(t_{i j}\right)=X_{i}\left(t_{i j}\right)-X_{i}\left(t_{i(j-1)}\right), \\
\Delta t_{i j}=t_{i j}-t_{i(j-1)}, \quad t_{i 0}=0, \quad X_{i}\left(t_{i 0}\right)=0 .
\end{gathered}
$$


From (13), the joint density can be obtained as

$$
\begin{aligned}
f\left(\Delta X_{i}\right) & \\
= & \frac{\Gamma(\beta+m / 2)}{(2 \pi \alpha)^{m / 2}|A|^{1 / 2} \Gamma(\beta)} \\
& \times\left(1+\frac{1}{2 \alpha}\left(\Delta X_{i}-\theta \Delta t_{i}\right)^{\prime} A^{-1}\left(\Delta X_{i}-\theta \Delta t_{i}\right)\right)^{-\beta-(m / 2)},
\end{aligned}
$$

where

$$
\begin{aligned}
\Delta X_{i} & =\left(\Delta X_{i}\left(t_{i 1}\right), \Delta X_{i}\left(t_{i 2}\right), \ldots, \Delta X_{i}\left(t_{i m}\right)\right), \\
\Delta t_{i} & =\left(\Delta t_{i 1}, \Delta t_{i 2}, \ldots, \Delta t_{i m}\right), \\
{\left[A_{p q}\right] } & = \begin{cases}\lambda \Delta t_{i p}^{2}+\Delta t_{i p}, & p=q, \\
\lambda \Delta t_{i p} \Delta t_{i q}, & p \neq q .\end{cases}
\end{aligned}
$$

Due to the independence assumption of the degradation measurements of different product, the log-likelihood function can be expressed as

$$
\begin{aligned}
& l(\alpha, \beta, \lambda, \theta \mid \Delta X) \\
& =\sum_{i=1}^{n}\left\{\log \Gamma\left(\beta+\frac{m}{2}\right)-\log \Gamma(\beta)-\frac{m}{2} \log 2 \pi \alpha-\frac{1}{2} \log |A|\right. \\
& \quad-\left(\beta+\frac{m}{2}\right) \\
& \left.\quad \times \log \left(1+\frac{1}{2 \alpha}\left(\Delta X_{i}-\theta \Delta t_{i}\right)^{\prime} A^{-1}\left(\Delta X_{i}-\theta \Delta t_{i}\right)\right)\right\} .
\end{aligned}
$$

Considering that the log-likelihood function is very complicated, the MCMC method is used to estimate the unknown parameters. In MCMC method, the prior distribution functions should be chosen carefully so that they are easily realistic. In this paper, the prior distribution of the four unknown parameters is assumed as

$$
\begin{gathered}
\theta \sim N\left(\mu_{0}, \sigma_{0}^{2}\right), \quad \alpha \sim \operatorname{Gamma}\left(\alpha_{01}, \alpha_{02}\right), \\
\beta \sim \operatorname{Gamma}\left(\beta_{01}, \beta_{02}\right), \quad \lambda \sim \operatorname{Gamma}\left(\lambda_{01}, \lambda_{02}\right),
\end{gathered}
$$

where $\mu_{0}, \sigma_{0}^{2}, \alpha_{01}, \alpha_{02}, \beta_{01}, \beta_{02}, \lambda_{01}$, and $\lambda_{02}$ are known hyperparameters.

Let $\pi(\cdot)$ denote the prior or posterior distributions for the parameters; by using Bayesian theory, the joint posterior distribution can be expressed as

$$
\begin{gathered}
\pi(\alpha, \beta, \lambda, \theta \mid \Delta X) \propto l(\alpha, \beta, \lambda, \theta \mid \Delta X) \pi\left(\theta \mid \mu_{0}, \sigma_{0}^{2}\right) \\
\quad \times \pi\left(\lambda \mid \lambda_{01}, \lambda_{02}\right) \pi\left(\alpha \mid \alpha_{01}, \alpha_{02}\right) \pi\left(\beta \mid \beta_{01}, \beta_{02}\right),
\end{gathered}
$$

where " $\propto$ " represents proportionality.

By using MCMC with the Gibbs sampling, the unknown parameters $\beta, \alpha, \theta$, and $\lambda$ can be obtained, and the Bayesian software package WinBUGS can easily carry out the Gibbs sampling (see in [18]).

\section{Individual Degradation Modeling and Reliability Assessment}

As far as the individual is concerned, in order to make effective maintenance decision, it is necessary to obtain the individual reliability assessment. But the individual units do not typically follow population-based model, and the reliability is apparent difference between the population and individual unit. In this section, we provide a Bayesian framework for the individual reliability assessment by integrating the population degradation information and the individual degradation data.

4.1. Updating Unknown Parameters. Suppose that the history degradation data of a particular unit is observed as $X\left(t_{0}\right)$, $X\left(t_{1}\right), \ldots, X\left(t_{k}\right)$; let

$$
\Delta X\left(t_{i}\right)=X\left(t_{i}\right)-X\left(t_{i-1}\right), \quad t_{0}=0, i=1,2, \ldots, k .
$$

According to the independent increment property of the Wiener process, we can get the following:

$$
\Delta X\left(t_{i}\right) \mid \mu, v \sim N\left(\mu \Delta t_{i}, v^{-1} \Delta t_{i}\right) .
$$

Then, we can get the PDF of $\Delta X\left(t_{i}\right)$ as follows:

$$
g\left(\Delta X\left(t_{i}\right) \mid \mu, v\right)=\frac{v^{1 / 2}}{\sqrt{2 \pi \Delta t_{i}}} \exp \left(-\frac{v\left(\Delta X\left(t_{i}\right)-\mu \Delta t_{i}\right)^{2}}{2 \Delta t_{i}}\right) .
$$

Let $\Delta X=\left(\Delta X_{1}, \Delta X_{2}, \ldots, \Delta X_{k}\right) ;$ according to the Bayesian method, the joint posterior PDF can be formulated as

$$
\pi(\mu, v \mid \Delta X)=\frac{\pi(\mu, v) L(\Delta X \mid \mu, v)}{\int_{0}^{+\infty} \int_{-\infty}^{+\infty} \pi(\mu, v) L(\Delta X \mid \mu, v) d \mu d v},
$$

where $L(\Delta X \mid \mu, v)=\prod_{i=1}^{k} g\left(\Delta X\left(t_{i}\right) \mid \mu, v\right)$ is the joint distribution, $\pi(\mu, v)$ is the joint prior distribution, and $\pi(\mu, v)=$ $\left(\alpha^{\beta} / \Gamma(\beta)\right) v^{\beta-1} \exp (-\alpha v)\left(v^{1 / 2} / \sqrt{2 \pi \lambda}\right) \exp \left(-\left(v(\mu-\theta)^{2} / 2 \lambda\right)\right)$.

Therefore, the above equation can be abbreviated:

$$
\begin{gathered}
\pi(\mu, v \mid \Delta X) \propto \pi(\mu, v) L(\Delta X \mid \mu, v) \\
\propto v^{\beta+k / 2-1} \exp \left(-v\left(\alpha+C-\frac{B^{2}}{4 A}\right)\right) \\
\times v^{1 / 2} \exp \left(-v A\left(\mu-\frac{B}{2 A}\right)^{2}\right) \\
\propto v^{\beta^{\prime}-1} \exp \left(-\alpha^{\prime} v\right) v^{1 / 2} \exp \left(-\frac{v\left(\mu-\theta^{\prime}\right)^{2}}{2 \lambda^{\prime}}\right),
\end{gathered}
$$


TABLE 1: Parameter estimation results under model $M_{2}$.

\begin{tabular}{lcccc}
\hline Parameter & Mean & Standard error & MC error & 95\% HPD interval \\
\hline$\alpha$ & 0.000346 & $1.435 E-4$ & $7.612 E-6$ & $(1.08 E-4,6.29 E-4)$ \\
$\beta$ & 3.477000 & 0.879800 & 0.049210 & $(2.31700,5.27300)$ \\
$\lambda$ & 0.001539 & 0.003153 & $1.651 E-4$ & $(1.61 E-6,1.14 E-2)$ \\
$\theta$ & 0.001965 & $5.386 E-5$ & $8.879 E-7$ & $(1.86 E-3,2.07 E-3)$ \\
\hline
\end{tabular}

TABLE 2: Parameter estimation results under model $M_{1}$.

\begin{tabular}{lcccc}
\hline Parameter & Mean & Standard error & MC error & 95\% HPD interval \\
\hline$\mu$ & 0.002054 & $5.446 E-5$ & $2.52 E-7$ & $(0.00194,0.00216)$ \\
$\sigma$ & 0.012870 & $5.933 E-4$ & $1.78 E-6$ & $(0.01177,0.01409)$ \\
\hline
\end{tabular}

where

$$
\begin{gathered}
\beta^{\prime}=\beta+\frac{k}{2}, \\
\alpha^{\prime}=\alpha+C-\frac{B^{2}}{4 A}, \\
\theta^{\prime}=\frac{B}{2 A}, \quad \lambda^{\prime}=\frac{1}{2 A}, \\
A=\frac{1}{2 \lambda}+\sum_{i=1}^{k} \frac{\Delta t_{i}}{2}, \\
B=\frac{\theta}{\lambda}+\sum_{i=1}^{k} \Delta X\left(t_{i}\right), \\
C=\frac{\theta^{2}}{2 \lambda}+\sum_{i=1}^{k} \frac{\Delta X^{2}\left(t_{i}\right)}{2 \Delta t_{i}} .
\end{gathered}
$$

Then, we can conclude that the joint posterior PDF is a normal-Gamma distribution with the following distribution as

$$
\pi(\mu, v \mid \Delta X)=N-\operatorname{Gamma}\left(\mu, v \mid \alpha^{\prime}, \beta^{\prime}, \theta^{\prime}, \lambda^{\prime}\right) .
$$

So, the Bayesian updated estimator of the unknown parameters $\mu$ and $v$ can be obtained as

$$
\begin{aligned}
\widehat{\mu}=\theta^{\prime}=\frac{B}{2 A} & =\frac{\theta / \lambda+\sum_{i=1}^{k} \Delta X\left(t_{i}\right)}{1 / \lambda+\sum_{i=1}^{k} \Delta t_{i}}=\frac{\theta / \lambda+X\left(t_{k}\right)}{1 / \lambda+t_{k}} \\
\widehat{v}=\widehat{\sigma}^{-2} & \\
= & \left(\beta+\frac{k}{2}\right) \\
& \times\left(\alpha+\frac{\theta^{2}}{2 \lambda}+\sum_{i=1}^{k} \frac{\Delta X^{2}\left(t_{i}\right)}{2 \Delta t_{i}}\right. \\
& \left.\quad \frac{\left((\theta / \lambda)+X\left(t_{k}\right)\right)^{2}}{\left((2 / \lambda)+2 t_{k}\right)}\right)^{-1}
\end{aligned}
$$

4.2. Obtaining RUL Distribution and Reliability Evaluation. Suppose that the degradation measurement data for a particular unit is $X\left(t_{k}\right)$ at time $t_{k}$. From the definition of the lifetime $T$, the RUL $R$ of the particular unit at time $t_{k}$ can be expressed as

$$
R=\inf \left\{t: X\left(t+t_{k}\right) \geq \xi\right\} .
$$

From $[14,21]$, we know that the key for estimating the RUL is to derive the PDF of lifetime. According to the independent increments property of the Wiener process, we can get

$$
\begin{aligned}
R & =\inf \left\{t: X\left(t+t_{k}\right) \geq \xi\right\} \\
& =\inf \left\{t: X\left(t+t_{k}\right)-X\left(t_{k}\right) \geq \xi-X\left(t_{k}\right)\right\} \\
& =\inf \left\{t: X(t) \geq \xi-X\left(t_{k}\right)\right\} .
\end{aligned}
$$

Based on (7) and (30), given the values of $\mu$ and $v$, the PDF of RUL of the particular unit can be written as

$$
f_{R}(t \mid \widehat{\mu}, \widehat{v})=\frac{\xi-X\left(t_{k}\right)}{\sqrt{2 \pi \widehat{v}^{-1} t^{3}}} \exp \left(-\frac{\widehat{v}\left(\xi-X\left(t_{k}\right)-\widehat{\mu} t\right)^{2}}{2 t}\right) .
$$

Then, the reliability based on the updated parameters can be given as follows:

$$
\begin{aligned}
R(t)= & 1-F_{R}(t \mid \widehat{\mu}, \widehat{v})=1-\int_{0}^{t} f_{R}(x \mid \widehat{\mu}, \widehat{v}) d x \\
= & \Phi\left(-\frac{\widehat{\mu} t-\left(\xi-X\left(t_{k}\right)\right)}{\sqrt{\widehat{v}^{-1} t}}\right)-\exp \left(\frac{2 \widehat{\mu}\left(\xi-X\left(t_{k}\right)\right)}{\widehat{v}^{-2}}\right) \\
& \times \Phi\left(-\frac{\xi-X\left(t_{k}\right)+\widehat{\mu} t}{\sqrt{\widehat{v}^{-1} t}}\right) .
\end{aligned}
$$

\section{Numerical Example}

In this section, a numerical example of laser device is given to demonstrate the validity of the proposed method. Operating current is an important performance for a laser device. Usually when the operating current decreases to a predefined threshold level, the laser device is considered to be failed. Fifteen laser devices were tested, and their operating current changed over time as shown in Figure 1, where horizontal axis represents the inspection time, while vertical axis represents the increase in operating current. The inspection interval is 250 hours, and the experiment was terminated at 4000 hours. Similar to $[12,14]$, we set the threshold as $\xi=10$. 
TABLe 3: Parameter estimation results under model $M_{3}$.

\begin{tabular}{lcccc}
\hline Parameter & Mean & Standard error & MC error & 95\% HPD interval \\
\hline$\eta$ & 0.002053 & $5.503 E-5$ & $2.352 E-7$ & $(1.95 E-3,2.16 E-3)$ \\
$\sigma_{\eta}$ & 0.000391 & $2.40 E-6$ & $2.56 E-8$ & $(3.86 E-4,3.96 E-4)$ \\
$\sigma$ & 0.011440 & $6.66 E-4$ & $9.85 E-6$ & $(1.02 E-2,1.28 E-2)$ \\
\hline
\end{tabular}

TABLE 4: Comparison of three degradation models.

\begin{tabular}{lccc}
\hline Model & $M_{1}$ & $M_{2}$ & $M_{3}$ \\
\hline DIC & -71.42 & -82.7 & -76.23 \\
Ranking & 3 & 1 & 2 \\
\hline
\end{tabular}

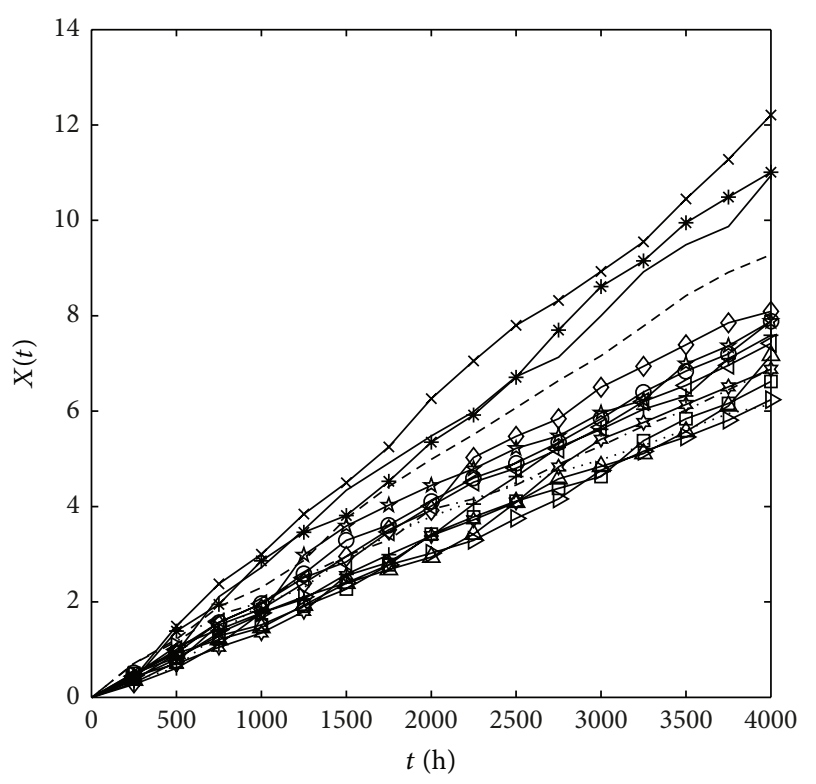

Figure 1: Degradation paths.

5.1. Population Reliability Assessment. In this subsection, we use the lasers data to estimate the reliability according to (8), (10), and (12) under the different degradation models $M_{1}, M_{2}$, and $M_{3}$, respectively.

For the degradation model $M_{2}$, the prior distributions are given based on experience as $\theta \sim N(0,0.001), \alpha \sim$ $\operatorname{Gamma}(0.00002,0.01), \beta \sim \operatorname{Gamma}(0.003,0.001), \lambda \sim$ $\operatorname{Gamma}(0.00001,0.01)$.

By using MCMC method, we generate 60,000 samples. A burn-in of 10,000 samples is used, with an additional 50,000 Gibbs samples used to estimate parameters. Table 1 tabulates posterior summaries, including parameters posterior mean, standard error, Monte Carlo error, and 95\% HPD interval. Similarly, the parameter estimation under models $M_{1}$ and $M_{3}$ can be obtained in Tables 2 and 3 .

To compare the three degradation models, the DIC criterion is adopted for model selection, and we calculate the criterion value under different degradation models in Table 4.

As shown in Table 4, comparing the fitting results from the different degradation models, we can find that the best fitting degradation model is the model $M_{2}$ with the lowest

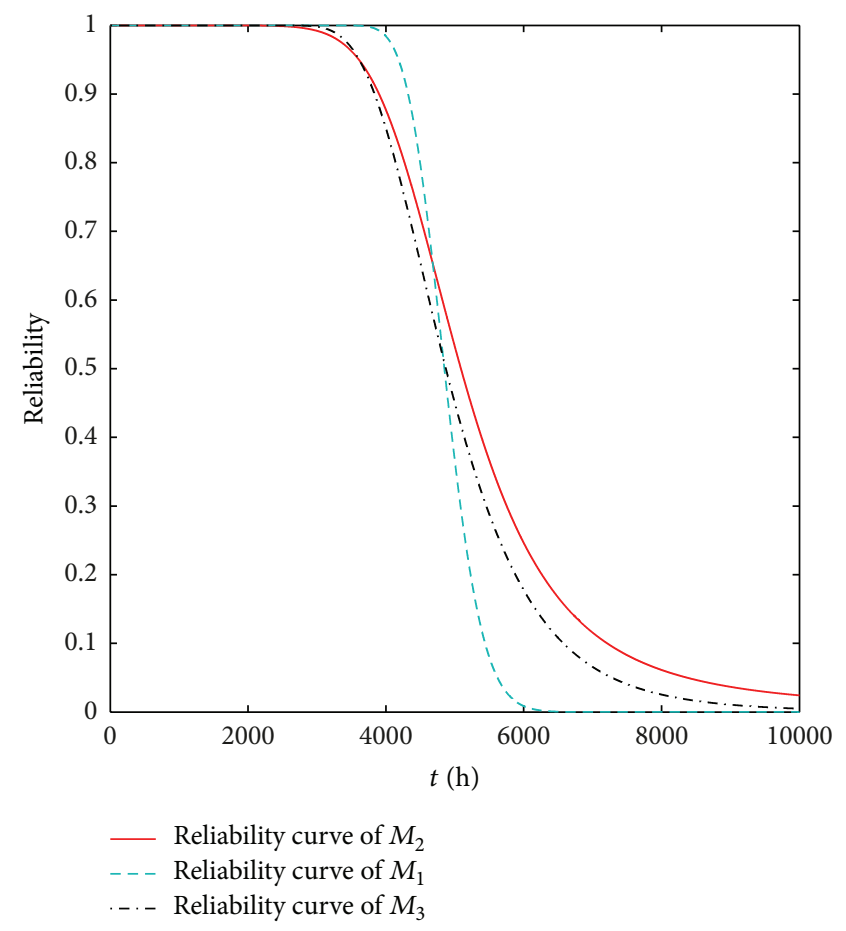

FIgURE 2: The reliability curve of three degradation models.

values of DIC. Correspondingly, we obtain the reliability curves under the models $M_{1}, M_{2}$, and $M_{3}$, respectively, as shown in Figure 2. From Figure 2, we can find that the reliability under the degradation model $M_{1}$ is not falling before the $3750 \mathrm{~h}$. But in fact, when the running time is arrived at at $3750 \mathrm{~h}$, some units have failed and the other units are gradually close to fail, so that the degradation model $M_{1}$ is the worst model to fit the actual degradation data. At the same time, the changing trend of the reliability curve under the degradation models $M_{2}$ and $M_{3}$ is nearly the same, and the reliability under the model $M_{2}$ is slightly conservative compared to the degradation model $M_{3}$.

5.2. Individual Reliability Assessment. The reliability analysis presented above provides a basis for the population performance reliability, but the individual unit may not typically follow the above models. We will use the Bayesian updated method to obtain the reliability evaluation of a particular individual degradation unit. The degradation history of the particular laser device is given in Table 5.

Firstly, we get the updated parameters about the particular laser device, and Table 6 shows the updating parameters at four different degradation time points. Once the parameters 
TABLE 5: Degradation history of a particular laser device.

\begin{tabular}{|c|c|c|c|c|c|c|c|c|c|c|c|c|c|c|c|c|}
\hline$t$ (hour) & 250 & 500 & 750 & 1000 & 1250 & 1500 & 1750 & 2000 & 2250 & 2500 & 2750 & 3000 & 3250 & 3500 & 3750 & 4000 \\
\hline$X(t)$ & 0.45 & 0.96 & 1.57 & 2.01 & 2.59 & 3.15 & 3.63 & 4.16 & 4.66 & 5.15 & 5.63 & 6.14 & 6.64 & 7.14 & 7.60 & 8.15 \\
\hline
\end{tabular}

TABLE 6: Updating parameters at different time for particular laser device.

\begin{tabular}{lcccc}
\hline$t$ (hour) & 250 & 1000 & 2000 & 3000 \\
\hline$\widehat{\mu}$ & $1.919 E-3$ & $1.992 E-3$ & $2.052 E-3$ & $2.032 E-3$ \\
$\widehat{\sigma}^{2}$ & $8.76 E-5$ & $6.99 E-5$ & $5.38 E-5$ & $4.27 E-5$ \\
\hline
\end{tabular}

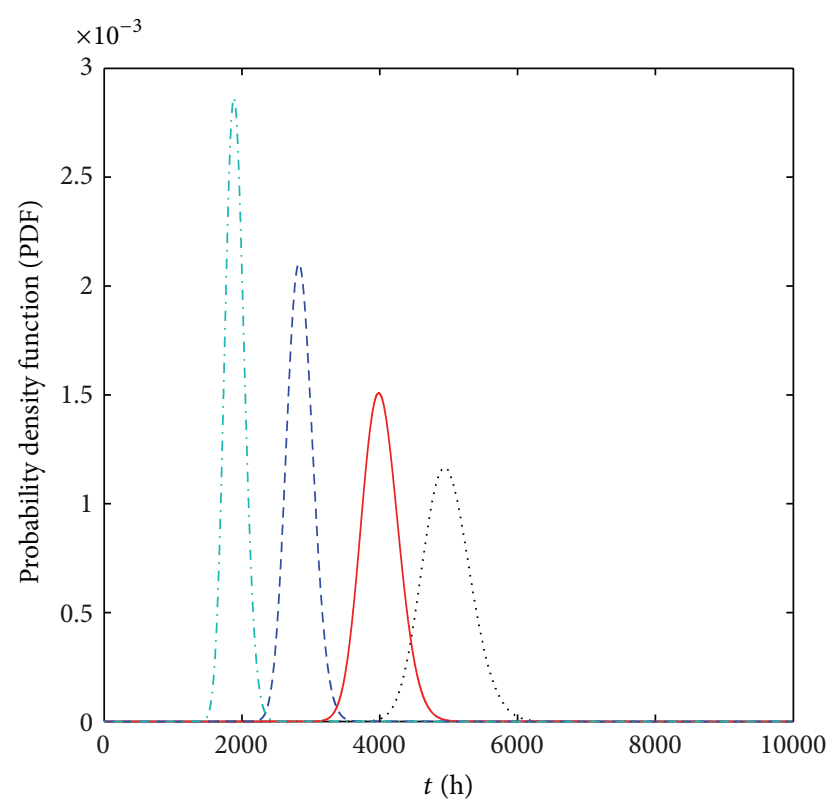

... The PDF curve of updating to $250 \mathrm{~h}$

- The PDF curve of updating to $1000 \mathrm{~h}$

- - - The PDF curve of updating to $2000 \mathrm{~h}$

The PDF curve of updating to $3000 \mathrm{~h}$

Figure 3: The PDF of the RUL at four different updating times.

in the model are updated, the PDF of the estimated RUL can be calculated at each time point.

Figure 3 illustrates the PDF of RUL distributions at four different time points. As shown in Figure 3, from right to left, we can find that the estimated PDF of the RUL becomes sharp, so that its uncertainty becomes smaller when the degradation parameters are updated. This implies that the uncertainty of the estimated RUL is reduced since more data are utilized during estimating the model parameters.

Figure 4 is the reliability curves of the particular laser device under the different updated time points. From Figure 4, we know that the reliability of the particular item changes regularly at different updating points. With the measurement time increasing, the reliability of the particular item decreases rapidly with time, which in turn shows the importance of adaptive evaluation once new degradation

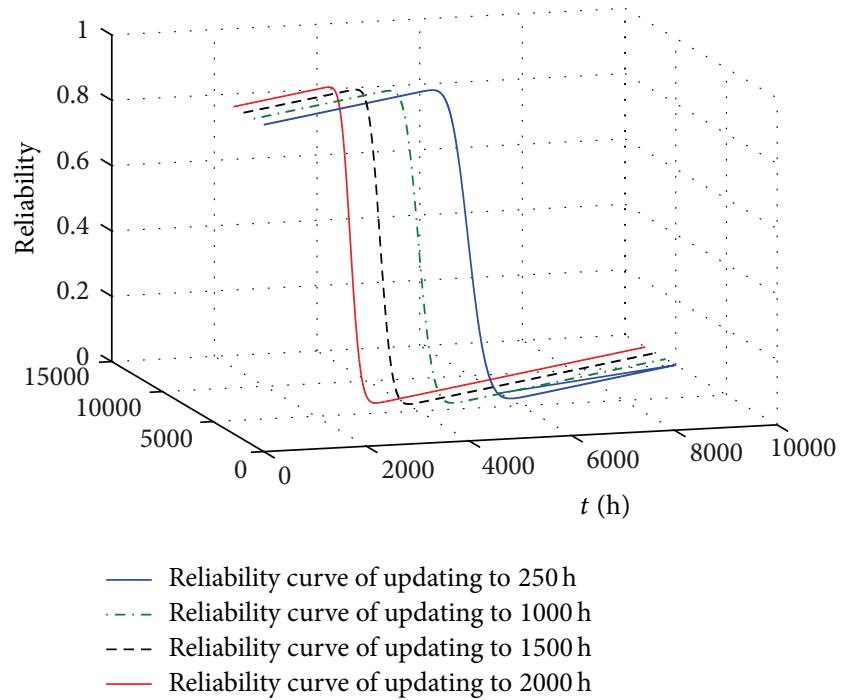

FIGURE 4: The performance reliability of particular laser device.

information is available. So, instead of the average population's characteristics, the reliability of particular item at different updating time points is very useful for making effective maintenance decision according to its own characteristic.

\section{Conclusions}

In this paper, a reliability evaluation framework consists of the population degradation modeling, the individual degradation modeling, the PDF of the RUL, and the reliability evaluation of individual unit. Our proposed solution combines several statistical tools, including model selection criteria, total probability law, Bayesian inference, and degradation modeling. A case study of the lasers data is given to validate the effectiveness of the proposed model and method. Main conclusions are as follows.

(1) The population and individual reliability assessment are discussed, and a Bayesian approach is proposed to integrate the population reliability information and the individual degradation data.

(2) A Wiener process with a random effect model is suitable for characterizing the capacity of the population degradation. And the individual degradation characteristic is obviously different from the population degradation characteristic.

(3) Because the likelihood function has very complicated expression, instead of directly maximizing the likelihood function, the MCMC method is used to estimate the unknown parameter. 
(4) Bayesian method can fully utilize the product history degradation data and individual performance degradation data, and it can be used for the reliability assessment for the individual degradation production.

(5) With the accumulation of the individual performance degradation data, the uncertainty of the estimated PDF of the RUL is reduced.

\section{Conflict of Interests}

The authors declare that there is no conflict of interests in this paper.

\section{Acknowledgments}

The authors would like to thank the anonymous reviewers for their useful comments which have greatly improved the paper. This work is supported by the National Natural Science Foundation of China (no. 50405021), the Fundamental Research Funds for the Central Universities and the Graduate Training Innovative Projects Foundation of Jiangsu Province (no. CXLX12_0081), and Youth Foundation of Hubei Educational Commission (no. Q20122603).

\section{References}

[1] W. Q. Meeker and L. A. Escobar, Statistical Method For Reliability Data, John Wiley \& Sons, New York, NY, USA, 1998.

[2] W. Nelson, Accelerated Testing: Statistical Models, Test Plans, and Data Analysis, John Wiley \& Sons, New York, NY, USA, 1990.

[3] M. J. Zuo, R. Y. Jiang, and R. C. M. Yam, "Approaches for reliability modeling of continuous state devices," IEEE Transactions on Reliability, vol. 48, no. 1, pp. 9-18, 1999.

[4] S.-T. Tseng, N. Balakrishnan, and C.-C. Tsai, "Optimal stepstress accelerated degradation test plan for gamma degradation processes," IEEE Transactions on Reliability, vol. 58, no. 4, pp. 611-618, 2009.

[5] Q. Duan, Z. Chen, and D. Zhao, "An expectation maximization algorithm to model failure times by continuous-time Markov chains," Mathematical Problems in Engineering, vol. 2010, Article ID 242567, 16 pages, 2010.

[6] H. W. Wang, J. Gao, and Z. Y. Liu, "Maintenance decision based on data fusion of aero engines," Mathematical Problems in Engineering, vol. 2013, Article ID 628792, 10 pages, 2013.

[7] C. Li and Y. Zhang, "Time-variant reliability assessment and its sensitivity analysis of cutting tool under invariant machining condition based on gamma process," Mathematical Problems in Engineering, vol. 2012, Article ID 676923, 19 pages, 2012.

[8] S.-T. Tseng, J. Tang, and I.-H. Ku, "Determination of burn-in parameters and residual life for highly reliable products," Naval Research Logistics, vol. 50, no. 1, pp. 1-14, 2003.

[9] J. Tang and T.-S. Su, "Estimating failure time distribution and its parameters based on intermediate data from a Wiener degradation model," Naval Research Logistics, vol. 55, no. 3, pp. 265-276, 2008.

[10] M.-Y. Lee and J. Tang, "A modified EM-algorithm for estimating the parameters of inverse Gaussian distribution based on timecensored Wiener degradation data," Statistica Sinica, vol. 17, no. 3, pp. 873-893, 2007.
[11] C. Park and W. J. Padgett, "Stochastic degradation models with several accelerating variables," IEEE Transactions on Reliability, vol. 55, no. 2, pp. 379-390, 2006.

[12] C.-Y. Peng and S.-T. Tseng, "Mis-specification analysis of linear degradation models," IEEE Transactions on Reliability, vol. 58, no. 3, pp. 444-455, 2009.

[13] X. Wang, "Wiener processes with random effects for degradation data," Journal of Multivariate Analysis, vol. 101, no. 2, pp. 340-351, 2010.

[14] X.-S. Si, W. Wang, C.-H. Hu, D.-H. Zhou, and M. G. Pecht, "Remaining useful life estimation based on a nonlinear diffusion degradation process," IEEE Transactions on Reliability, vol. 61, no. 1, pp. 50-67, 2012.

[15] N. Z. Gebraeel, M. A. Lawley, R. Li, and J. K. Ryan, "Residual-life distributions from component degradation signals: a Bayesian approach," IIE Transactions, vol. 37, no. 6, pp. 543-557, 2005.

[16] N. Z. Gebraeel and M. A. Lawley, "A neural network degradation model for computing and updating residual life distributions," IEEE Transactions on Automation Science and Engineering, vol. 5, no. 1, pp. 154-163, 2008.

[17] L. Bian and N. Gebraeel, "Computing and updating the firstpassage time distribution for randomly evolving degradation signals," IIE Transactions, vol. 44, pp. 974-987, 2012.

[18] I. Ntzoufras, Bayesian Modeling Using WinBUGS, John Wiley \& Sons, Hoboken, NJ, USA, 2009.

[19] D. J. Spiegelhalter, N. G. Best, B. P. Carlin, and A. van der Linde, "Bayesian measures of model complexity and fit," Journal of the Royal Statistical Society B, vol. 64, no. 4, pp. 583-639, 2002.

[20] C. A. Santos and J. A. Achcar, "A Bayesian analysis in the presence of covariates for multivariate survival data: an example of application," Revista Colombiana de Estadística, vol. 34, no. 1, pp. 111-131, 2011.

[21] W. Wang, M. Carr, W. Xu, and K. Kobbacy, "A model for residual life prediction based on Brownian motion with an adaptive drift," Microelectronics Reliability, vol. 51, no. 2, pp. 285-293, 2011. 


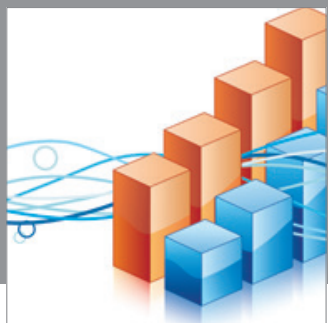

Advances in

Operations Research

mansans

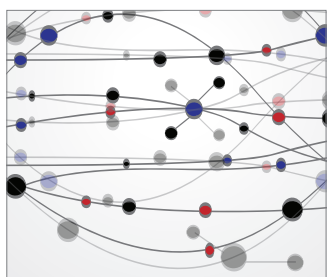

The Scientific World Journal
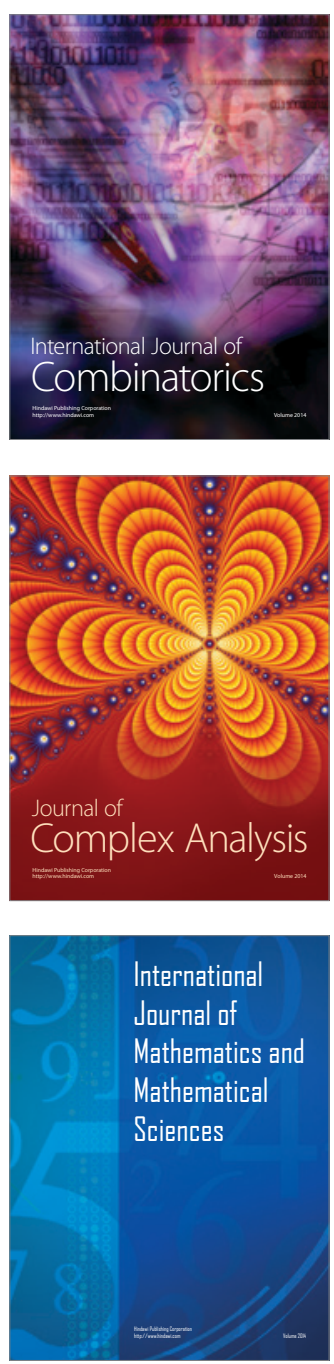
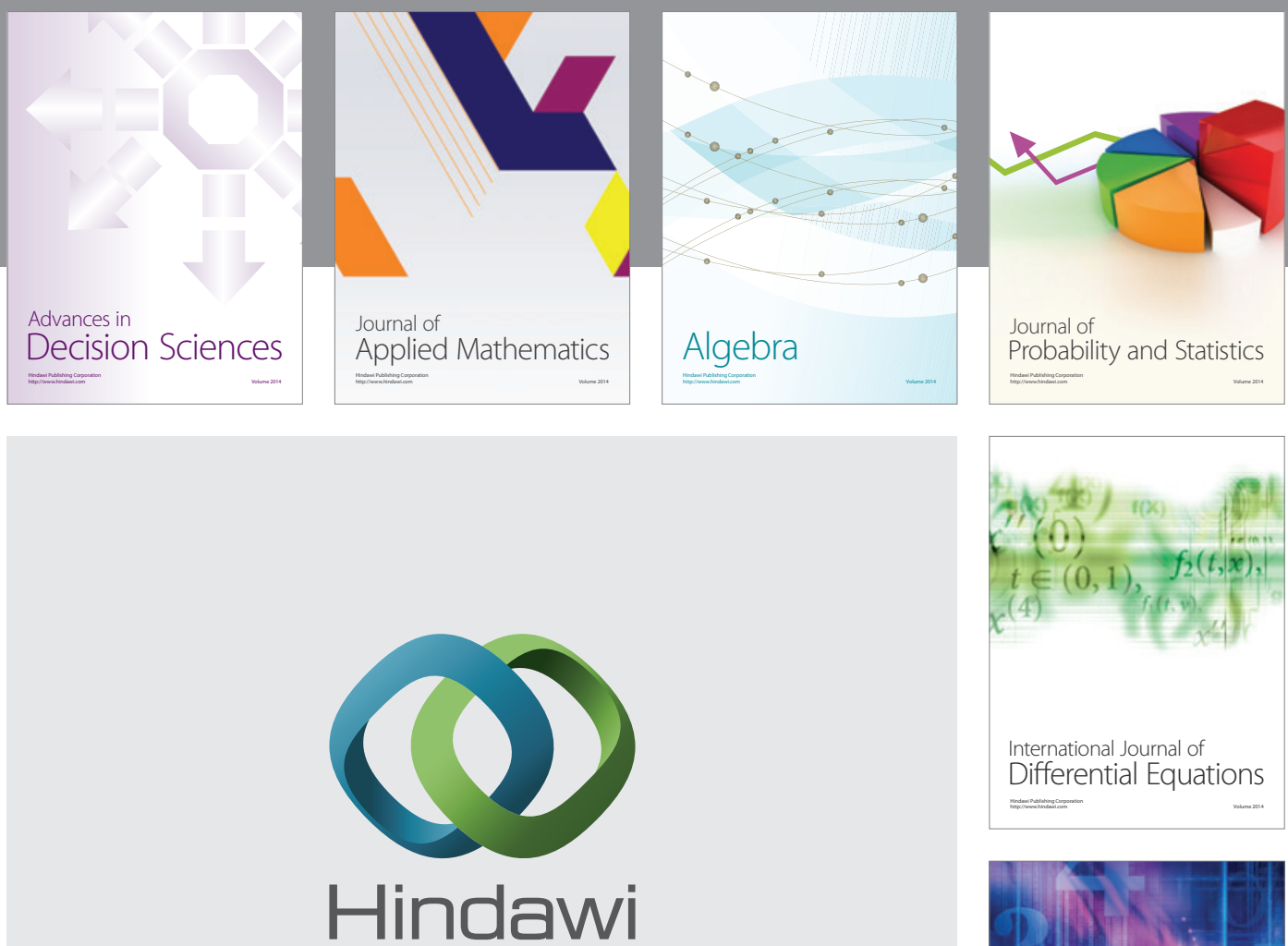

Submit your manuscripts at http://www.hindawi.com
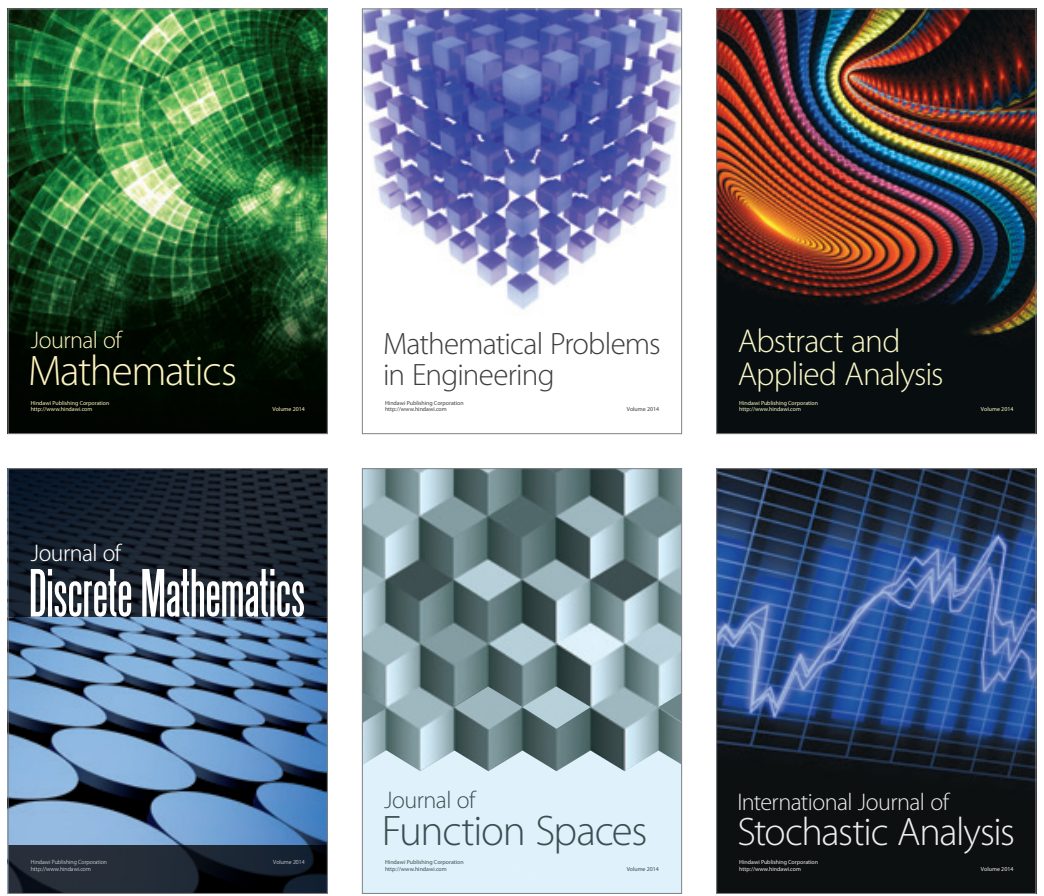

Journal of

Function Spaces

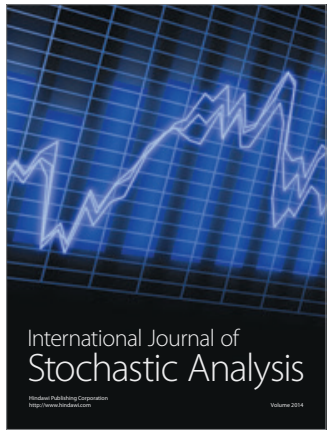

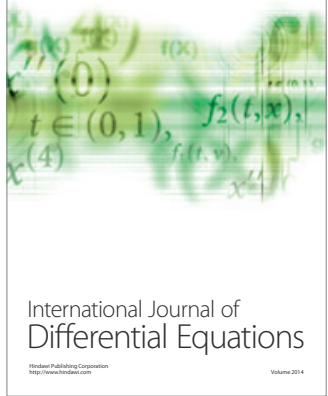
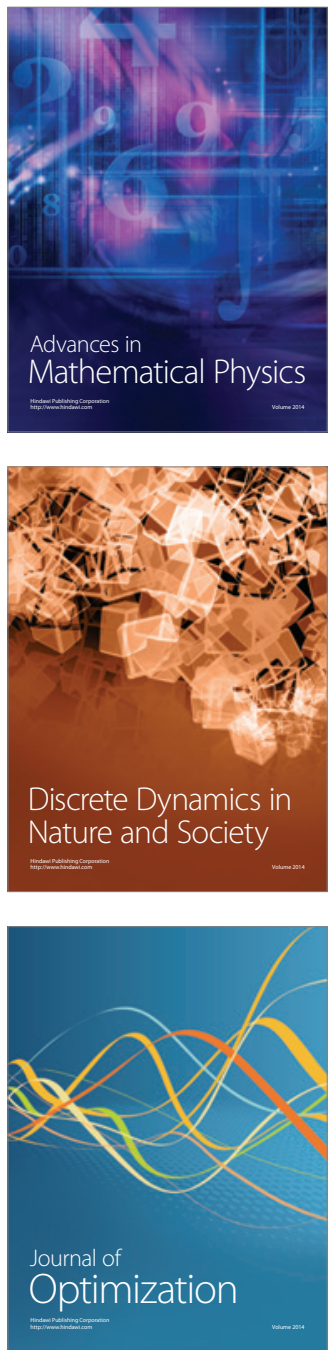